\title{
651.
}

\section{ON A SPECIAL SURFACE OF MINIMUM AREA.}

[From the Quarterly Journal of Pure and Applied Mathematics, vol. xIV. (1877), pp. 190-196.]

A VERY remarkable form of the surface of minimum area was obtained by Prof. Schwarz in his memoir "Bestimmung einer speciellen Minimal-fläche," Berlin, 1871, [Ges. Werke, t. I., pp. 6-125], crowned by the Academy of Sciences at Berlin. The equation of the surface is

$$
1+\mu \nu+\nu \lambda+\lambda \mu=0
$$

where $\lambda, \mu, \nu$ are functions of $x, y, z$ respectively, viz.

$$
x=-\int_{\lambda}^{\infty} \frac{d \theta}{\sqrt{\left(\frac{3}{4} \theta^{4}+\frac{5}{2} \theta^{2}+\frac{3}{4}\right)}},
$$

and $y, z$ are the same functions of $\mu, \nu$ respectively. A direct verification of the theorem that this is a surface of minimum area, satisfying, that is, the differential equation

$$
r\left(1+q^{2}\right)-2 p q s+t\left(1+p^{2}\right)=0,
$$

is given in the memoir; but the investigation may be conducted in quite a different manner, so as to be at once symmetrical and somewhat more general, viz. we may enquire whether there exists a surface of minimum area

$$
1+\mu \nu+\nu \lambda+\lambda \mu=0
$$

where the determining equations are

$$
\begin{aligned}
& \lambda^{\prime 2}=a \lambda^{4}+b \lambda^{2}+c, \\
& \mu^{\prime 2}=a \mu^{4}+b \mu^{2}+c, \\
& \nu^{\prime 2}=a \nu^{4}+b \nu^{2}+c,
\end{aligned}
$$


$\left(\lambda^{\prime}=\frac{d \lambda}{d x}, \& c.\right)$. I find that the coefficients $a, b, c$ must satisfy four homogeneous quadric equations, which, in fact, admit of simultaneous solution, and that in three distinct ways; viz. assuming $a=1$, the solutions are

that is,

$$
\begin{aligned}
& a=1, \quad b=\frac{10}{3}, \quad c=1, \\
& a=1, \quad b=-2, c=1 \text {, } \\
& a=1, \quad b=-\frac{3}{2}, \quad c=-\frac{1}{3} \text {; }
\end{aligned}
$$

$$
\lambda^{\prime 2}=\lambda^{4}+\frac{10}{3} \lambda^{2}+1\left\{=\frac{4}{3}\left(\frac{3}{4} \lambda^{4}+\frac{5}{2} \lambda^{2}+\frac{3}{4}\right)\right\},
$$

which gives Schwarz's surface:

$$
\lambda^{\prime 2}=\lambda^{4}-2 \lambda^{2}+1 \text { or } \lambda^{\prime}= \pm\left(\lambda^{2}-1\right)
$$

which, it is easy to see, gives only $x+y+z=$ const.; and

$$
\lambda^{\prime 2}=\lambda^{4}-\frac{2}{3} \lambda^{2}-\frac{1}{3}, \quad=\left(\lambda^{2}-1\right)\left(\lambda^{2}+\frac{1}{3}\right),
$$

which is a surface similar in its nature to Schwarz's surface.

The investigation is as follows: the condition to be satisfied by a surface of minimum area $U=0$ is

$$
(\mathrm{a}+\mathrm{b}+\mathrm{c})\left(X^{2}+Y^{2}+Z^{2}\right)-(\mathrm{a}, \mathrm{b}, \mathrm{c}, \mathrm{f}, \mathrm{g}, \mathrm{h} \gamma X, Y, Z)^{2}=0
$$

where $(X, Y, Z)$ are the first derived coefficients and (a, b, c, f, g, h) the second derived coefficients of $U$ in regard to the coordinates. Considering $U$ as a function of $\lambda, \mu, \nu$, which are functions of $x, y, z$ respectively, and writing $(L, M, N)$ and $(a, b, c, f, g, h)$ for the first and second derived functions of $U$ in regard to $\lambda, \mu, \nu$, also $\lambda^{\prime}, \lambda^{\prime \prime}$ for the first and second derived functions of $\lambda$ in regard to $x$, and so for $\mu^{\prime}, \mu^{\prime \prime}$ and $\nu^{\prime}, \nu^{\prime \prime}$ : we have

$$
\begin{gathered}
(X, Y, Z)=\left(L \lambda^{\prime}, M \mu^{\prime}, N \nu^{\prime}\right), \\
(\mathrm{a}, \mathrm{b}, \mathrm{c}, \mathrm{f}, \mathrm{g}, \mathrm{h})=\left(a \lambda^{\prime 2}+L \lambda^{\prime \prime}, b \mu^{\prime 2}+M \mu^{\prime \prime}, c \nu^{\prime 2}+N \nu^{\prime \prime}, f \mu^{\prime} \nu^{\prime}, g \nu^{\prime} \lambda^{\prime}, h \lambda^{\prime} \mu^{\prime}\right),
\end{gathered}
$$

and for the particular surface $U=1+\mu \nu+\nu \lambda+\lambda \mu=0$, the values are

$$
(L, M, N, a, b, c, f, g, h)=(\mu+\nu, \nu+\lambda, \lambda+\mu, 0,0,0,1,1,1) .
$$

Hence the condition is found to be

$$
\begin{aligned}
& 2 \mu^{\prime 2} \nu^{\prime 2}(\lambda+\mu)(\lambda+\nu) \\
+ & 2 \nu^{\prime 2} \lambda^{\prime 2}(\mu+\nu)(\mu+\lambda) \\
+ & 2 \lambda^{\prime 2} \mu^{\prime 2}(\nu+\lambda)(\nu+\mu) \\
- & \lambda^{\prime \prime}(\mu+\nu)\left\{(\lambda+\nu)^{2} \mu^{\prime 2}+(\lambda+\mu)^{2} \nu^{\prime 2}\right\} \\
- & \mu^{\prime \prime}(\nu+\lambda)\left\{(\mu+\lambda)^{2} \nu^{\prime 2}+(\mu+\nu)^{2} \lambda^{\prime 2}\right\} \\
- & \nu^{\prime \prime}(\lambda+\mu)\left\{(\nu+\mu)^{2} \lambda^{\prime 2}+(\nu+\lambda)^{2} \mu^{\prime 2}\right\}=0,
\end{aligned}
$$


or say this is

$$
\begin{aligned}
& 2 \Sigma \mu^{\prime 2} \nu^{\prime 2}(\lambda+\mu)(\lambda+\nu) \\
& -\Sigma \lambda^{\prime \prime}(\mu+\nu)\left\{(\lambda+\nu)^{2} \mu^{\prime 2}+(\lambda+\mu)^{2} \nu^{\prime 2}\right\}=0 .
\end{aligned}
$$

We have to write in this equation $\lambda^{\prime 2}=a \lambda^{4}+b \lambda^{2}+c$, and therefore $\lambda^{\prime \prime}=2 a \lambda^{3}+b \lambda$, \&c.; the left-hand side, call it $\Omega$, is a symmetrical function of $\lambda, \mu, \nu$, and is consequently expressible as a rational function of

$$
\begin{aligned}
& p,=\lambda+\mu+\nu, \\
& q,=\mu \nu+\nu \lambda+\lambda \mu, \\
& r,=\lambda \mu \nu .
\end{aligned}
$$

We ought to have $\Omega=0$, not identically, but in virtue of the equation $1+q=0$, that is, $\Omega$ should divide by $1+q$; or, what is the same thing, $\Omega$ should vanish on writing therein $q=-1$.

To effect the reduction as easily as possible, observe that we have $(\lambda+\mu)(\lambda+\nu)=\lambda^{2}+q$; and therefore

$$
\Sigma \mu^{\prime 2} \nu^{\prime 2}(\lambda+\mu)(\lambda+\nu)=\Sigma \lambda^{2} \mu^{\prime 2} \nu^{\prime 2}+q \Sigma \mu^{\prime 2} \nu^{\prime 2} .
$$

Similarly, in the second term,

$$
(\mu+\nu)(\lambda+\nu)^{2}=(\nu+\lambda)\left(\nu^{2}+q\right) \text { and }(\mu+\nu)(\lambda+\mu)^{2}=(\mu+\lambda)\left(\mu^{2}+q\right) .
$$

The complete value of $\Omega$ thus is

where

$$
\Omega=2(A q+B)-\left[(C+D) q+E+F^{\prime}\right]
$$

$$
\begin{array}{ll}
A=\Sigma \lambda^{2} \mu^{\prime 2} \nu^{\prime 2}, & B=\Sigma \mu^{\prime 2} \nu^{\prime 2}, \\
C=\Sigma \lambda \lambda^{\prime \prime}\left(\nu^{2} \mu^{\prime 2}+\mu^{2} \nu^{\prime 2}\right), & D=\Sigma \lambda^{\prime \prime}\left(\nu^{2} \mu^{\prime 2}+\mu^{2} \nu^{\prime 2}\right), \\
E=\Sigma \lambda \lambda^{\prime \prime}\left(\mu^{\prime 2}+\nu^{\prime 2}\right), & F=\Sigma \lambda^{\prime \prime}\left(\nu \mu^{\prime 2}+\mu \nu^{\prime 2}\right) .
\end{array}
$$

We find without difficulty

$$
\begin{aligned}
A= & a^{2}\left(q^{4}-4 q^{2} p r+4 q r^{2}+2 p^{2} r^{2}\right) \\
& +a b\left(-2 q^{3}+q^{2} p^{2}+4 q p r-3 r^{2}-2 p^{3} r\right) \\
& +a c\left(4 q^{2}-8 q p^{2}+8 p r+2 p^{4}\right) \\
& +b^{2}\left(q^{2}-2 p r\right) \\
& +b c\left(-4 q+2 p^{2}\right) \\
& +c^{2}(3) \\
B= & a^{2}\left(q^{2} r^{2}+2 p r^{3}\right) \\
& +a b\left(-4 q r^{2}+2 p^{2} r^{2}\right) \\
& +a c\left(-2 q^{3}+q^{2} p^{2}+4 q p r-3 r^{2}-2 p^{3} r\right) \\
& +b^{2}\left(3 r^{2}\right) \\
& +b c\left(2 q^{2}-4 p r\right) \\
& +c^{2}\left(-2 q+p^{2}\right),
\end{aligned}
$$

C. $\mathrm{X}$. 


$$
\begin{aligned}
C= & a^{2}\left(4 q^{4}-16 q^{2} p r+16 q r^{2}+8 p^{2} r^{2}\right) \\
& +a b\left(-6 q^{3}+3 q^{2} p^{2}+12 q p r-9 r^{2}-6 p^{3} r\right) \\
& +a c\left(8 q^{2}-16 q p^{2}+16 p r+4 p^{4}\right) \\
& +b^{2}\left(2 q^{2}-4 p r\right) \\
& +b c\left(-4 q+2 p^{2}\right) \\
D= & a^{2}\left(2 q^{2} p r-2 q r^{2}-4 p^{2} r^{2}\right) \\
& +a b\left(-4 q p r+2 p^{3} r\right) \\
& +a c\left(-4 q^{2}+2 q p^{2}-2 p r\right) \\
& +b^{2}(2 p r) \\
& +b c(2 q), \\
E= & +a^{2}\left(\quad 4 q^{2} r^{2}-8 p r^{3}\right) \\
& +a b\left(-12 q r^{2}+6 p^{2} r^{2}\right) \\
& +a c\left(-4 q^{3}+2 q^{2} p^{2}+8 q p r-6 r^{2}-4 p^{3} r\right) \\
& +b^{2}\left(\quad 6 r^{2}\right) \\
& +b c\left(\quad 2 q^{2}-4 p r\right), \\
F= & a^{2}\left(\quad 4 p r^{3}\right) \\
& +a b\left(\quad q^{2} p r+3 q r^{2}-2 p^{2} r^{2}\right) \\
& +a c\left(\quad 4 q^{3}-12 q p r+12 r^{2}\right) \\
& +b^{2}\left(\quad q p r-3 r^{2}\right) \\
& +b c\left(-2 q^{2}+q x^{2}-p r\right)
\end{aligned}
$$

where in each line the terms are arranged according to their order in $p, r$.

Substituting, we find

$$
\begin{aligned}
\Omega= & a^{2}\left(-2 q^{5}+6 q^{3} p r-8 q^{2} r^{2}\right) \\
& +a b\left(2 q^{4}-q^{3} p^{2}-q^{2} p r+4 q r^{2}\right) \\
& +a c\left(-2 q^{2} p^{2}+14 q p r-12 r^{2}\right) \\
& +b^{2}\left(-3 q p r+3 r^{2}\right) \\
& +b c\left(-2 q^{2}+q p^{2}-3 p r\right) \\
& +c^{2}\left(2 q+2 p^{2}\right)
\end{aligned}
$$

viz. writing $q=-1$, this is

$$
\begin{aligned}
& \Omega=a^{2}\left(2-6 p r-8 r^{2}\right) \\
& +a b\left(2+p^{2}-p r-4 r^{2}\right) \\
& +a c\left(-2 p^{2}-14 p r-12 r^{2}\right) \\
& +b^{2}\left(3 p r+3 r^{2}\right) \\
& +b c\left(-2-p^{2}-3 p r \quad\right) \\
& +c^{2}\left(-2-2 p^{2} \quad\right) \text {; }
\end{aligned}
$$


or, what is the same thing, it is

$$
\begin{aligned}
& =\left(\begin{array}{ll}
2 a^{2}+2 a b & \left.-2 b c-2 c^{2}\right)
\end{array}\right. \\
& +p^{2}\left(\quad a b-2 a c \quad-b c+2 c^{2}\right) \\
& +p r\left(-6 a^{2}-a b-14 a c+3 b^{2}-3 b c \quad\right) \\
& +r^{2}\left(-8 a^{2}-4 a b-12 a c+3 b^{2} \quad\right) \text {; }
\end{aligned}
$$

so that, writing for convenience $a=1$, the equations to be satisfied are

$$
\begin{array}{rr}
2-2 c^{2}+2(1-c) \quad b=0, \\
-2 c+2 c^{2}+(1-c) b=0, \\
-6-14 c+3 b^{2}-(1+3 c) \quad b=0, \\
-8-12 c+3 b^{2}-\quad 4 b=0 .
\end{array}
$$

The first and second are $(1-c)(2+2 c+2 b)=0$ and $(1-c)(-2 c+b)=0$; viz. they give $c=1$, or else $b=-\frac{2}{3}, c=\frac{1}{3}$. In the former case, the third and fourth equations each become $3 b^{2}-4 b-20=0$, that is $(3 b-10)(b-2)=0$; in the latter case, they are satisfied identically; hence we have for $a, b, c$ the three systems of values mentioned at the beginning.

This completes the investigation; but it is interesting to find the values assumed by the other factor of $\Omega$ on substituting therein for $a, b, c$ the foregoing several systems of values. We have in general

$$
\begin{aligned}
& \Omega=\quad-2 a^{2} q^{3}+2 a b q^{4}-2 b c q^{2}+2 c^{2} q \\
& +p^{2}\left(-a b q^{3}-2 a c q^{2}+b c q+2 c^{2} \quad\right) \\
& +\operatorname{pr}\left(6 a^{2} q^{3}-a b q^{2}+14 a c q-3 b^{2} q-3 b c\right) \\
& +r^{2}\left(-8 a^{2} q^{2}+4 a b q-12 a c+3 b^{2} \quad\right) \\
& =-2 a^{2}\left(q^{5}+1\right)+2 a b\left(q^{4}-1\right)-2 b c\left(q^{2}-1\right)+2 c^{2}(q+1) \\
& +p^{2}\left\{-a b\left(q^{3}+1\right)-2 a c\left(q^{2}-1\right)+b c(q+1)\right\} \\
& +p r\left\{6 a^{2}\left(q^{3}+1\right)-a b\left(q^{2}-1\right)+\left(14 a c-3 b^{2}\right)(q+1)\right\} \\
& \left.+r^{2} \quad-8 a^{2}\left(q^{2}-1\right)+4 a b(q+1)\right\}
\end{aligned}
$$

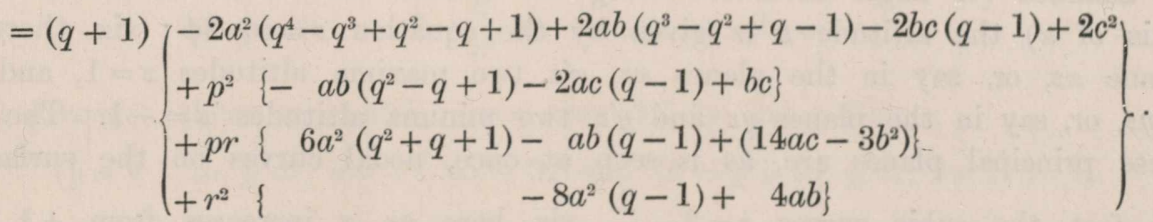

Hence writing, first, $a=c=1, b=\frac{10}{3}$, we obtain, after some reductions, $\Omega=(q+1)\left\{-2 q(\dot{q}-1)\left(q^{2}-\frac{10}{3} q+1\right)+p^{2}(q-1)\left(-\frac{10}{3} q-2\right)+p r\left(6 q^{2}-\frac{28}{3} q-10\right)+r^{2}-8 q+\frac{64}{3}\right\} ;$ secondly, writing $a=c=1, b=-2$, we obtain $\Omega=(q+1)\left\{-2(q+1)^{2}\left(q^{2}+1\right)+p^{2} \cdot 2(q-1)^{2}+2 p r\left(3 q^{2}-2 q+6\right)-8 r^{2} q\right\} ;$ and, thirdly, writing $a=1, b=-\frac{1}{3}, c=-\frac{2}{3}$, we obtain $\Omega=(q+1)\left\{\left(-2 q^{4}+\frac{4}{3} q^{3}-\frac{4}{3} q^{2}+\frac{8}{9} q\right)+p^{2}\left(-\frac{1}{3} q^{2}+\frac{5}{3} q-\frac{13}{9}\right)+p r\left(6 q^{2}-\frac{17}{3} q-\frac{10}{3}\right)+r^{2}\left(-8 q+\frac{20}{3}\right)\right\}$. 